\title{
Community acquired methicillin sensitive Staphylococcus aureus bacteremia, meningitis and brain abscess: A unique presentation
}

\author{
Carlos Gonzalez, Juan Roa, Nehad Shabarek
}

\begin{abstract}
Introduction: Staphylococcus aureus causing isolate central nervous system (CNS) infection is rare. It is mostly related to either neurosurgical intervention or previous local or systemic infection rather than spontaneous isolated infection in adults. Case Report: We report a case of a previously healthy 19-year-old male with no risk factors of Staphylococcus aureus infection, who was found to have methicillin sensitive Staphylococcus aureus (MSSA) bacteremia, meningitis and cerebral abscess, without an apparent source of infection. Conclusion: Our case is a unique presentation of a young male presenting with isolated CNS infection by MSSA. This case highlights the challenge of early diagnosis of brain abscess, an entity that presents very often with non-specific signs, the diagnosis of which can be easily missed, with repercussions on long-term disability and mortality.
\end{abstract}

Carlos Gonzalez 1 , Juan Roa ${ }^{2}$, Nehad Shabarek ${ }^{3}$

Affiliations: ${ }^{1}$ PGY-1 Internal Medicine, Lincoln Medical and Mental Health Center of Weill Medical College at Cornell University, New York, USA; 2 PGY-2 Internal Medicine, Lincoln Medical and Mental Health Center of Weill Medical College at Cornell University, New York, USA; ${ }^{3}$ Attending physian, associatted program director, Department of Internal Medicine, Lincoln Medical and Mental Health Center of Weill Medical College at Cornell University, New York, USA.

Corresponding Author: Carlos Gonzalez, MD Department of Internal Medicine, Lincoln Medical and Mental Health Center, 234 E. 149th, St \#8-32, Bronx, NY 10451; Ph: 7185794739; Fax: 7185794836; Email: carlosgonzalez0521@yahoo.com;

carlos.gonzalez.lengua@nychhc.org

Received: 08 April 2012

Accepted: 28 July 2012

Published: 01 January 2013
Keywords: Staphylococcus aureus, Bacteremia, Meningitis, Brain abscess

$$
* * * * * * * * *
$$

Gonzalez C, Roa J, Shabarek N. Community acquired methicillin sensitive Staphylococcus aureus bacteremia, meningitis and brain abscess: A unique presentation. International Journal of Case Reports and Images 2013;4(1):19-23.

$$
* * * * * * * * *
$$

doi:10.5348/ijcri-2013-01-251-CR-5

\section{INTRODUCTION}

Staphylococcus aureus is the most virulent of the staphylococcal species with unique versatility to cause various types of infections, from community acquired mild skin infections to highly lethal infections such as necrotizing pneumonia, endocarditis or central nervous system (CNS) infections. CNS infections caused by Staphylococcus aureus are in general uncommon. Various series report Staphylococcus aureus as the fourth or fifth most common etiology for neuroinfection; more commonly as cause of brain abscess and less commonly as an isolated cause of meningitis (less than $5 \%$ ) $[1-5,6]$. In either case, CNS infection caused by Staphylococcus aureus is related to recent neurosurgical intervention or associated with systemic or local infection classically described as a complication of endocarditis or soft tissue infection $[1-2,6]$. Although there are a few case reports of CNS infection in patients without an apparent source, most of them are related to community acquire methicillin resistant Staphylococcus aureus (CA-MRSA) infections [7]. To date, we have not found any reported case of confirmed meningitis with brain abscess, caused by MSSA infection in a previous healthy patient with no 
predisposing infection or risk factors. We report a case of MSSA bacteremia with meningitis and brain abscess in a previous healthy young male without any risk factors and no obvious source of infection, the detection of which was delayed due to a normal brain noncontrast computed tomography (CT) scan findings.

\section{CASE REPORT}

A 19-year-old Hispanic male student, presented to the emergency department (ED) complaining of dull frontal and occipital headache associated with fever, few episodes of vomiting, poor oral intake and myalgia for two days. His past medical history was only remarkable for a recent dental procedure. He denied any sick contacts and drug abuse. Initial physical examination was remarkable for tachycardia of $123 \mathrm{bpm}$ and temperature of $38^{\circ} \mathrm{C}$. No meningeal signs or focal deficits were found on the initial presentation. The remaining physical examination was unremarkable. Initial laboratory work up was positive for mild hyperglycemia with blood sugar $143 \mathrm{mg} / \mathrm{dL}$, Na 132 $\mathrm{mEq} / \mathrm{dL}$ and neutrophilia $92 \%$ with WBC count $11 \times 10^{3} / \mathrm{mm}^{3}$. Brain CT scan and lumbar puncture (LP) were performed to exclude meningitis or any intracranial pathology. Both the investigations were negative. The patient received symptomatic management with IV fluids and non- steroidal antiinflammatory drugs (NSAIDs) for fever, with notable improvement. He was discharged home from the ED with symptomatic management. One day after the initial presentation, blood cultures were reported positive for MSSA. Multiple attempts to contact the patient and his family were unfortunately unsuccessfully.

Four days after the initial presentation, the patient was brought to the ED again, after being found unresponsive. Patient's mother stated he was doing well until early morning when he complained of malaise, fever and headache. Physical examination was positive for temperature of $40.5^{\circ} \mathrm{C}$. Other vital signs were normal. The patient was lethargic, found to have nuchal rigidity and was only responsive to painful stimuli. Glasgow coma scale (GCS) was 9/15. Mild patchy folliculitis was noted in the suprapubic area with crusted lesions. He was intubated and transfered to the medical intensive care unit (MICU) with a diagnosis of possible meningitis and MSSA bacteremia based on his previous blood culture. Repeat LP and blood culture were performed. Lumbar puncture showed xanthochromia with $\mathrm{RBC} 11 / \mathrm{mm}^{3}$, WBC count $817 / \mathrm{mm}^{3}$ (neutrophils 90\%, lymphocytes 2\%, monocytes $8 \%$ ), glucose $28 \mathrm{mg} / \mathrm{dL}$ and protein $126 \mathrm{mg} / \mathrm{dL}$. Blood cultures grew MSSA with the same pattern of sensitivity as the first culture. Non-contrast brain CT scan was again performed, with normal findings (Figure 1). The patient was initiated on bacterial meningitis treatment with ceftriaxone $2 \mathrm{~g}$ q12hr, nafcillin $2 \mathrm{~g}$ q4hr, and dexamethazone $4 \mathrm{mg}$ q6hr. Ceftriaxone was switched to gentamicin $60 \mathrm{mg}$ qh8hr on day-2.

During the hospital course in MICU, transthoracic echocardiogram (TTE) was negative for endocarditis, urine toxicology was negative and HIV status was negative. The patient was extubated four days after admission, at which time a left palpebral ptsosis was noted. His pupils were symmetric, $3 \mathrm{~mm}$ in diameter, and reactive. He remained lethargic and a contrast enhanced magnetic resonance imaging (MRI) was ordered due to his lack of neurological improvement. The MRI scan of brain showed a focal lesion centered at the left anterior thalamus with contiguous extension into the subthalamic region and left cerebral peduncle of the left midbrain, with a small focus of contralateral extension into the right thalamus (left lesion measure: $3.5 \mathrm{~cm}$ height, $2 \mathrm{~cm}$ antero-posterior and 1.8 transverse, right lesion $0.4 \mathrm{~cm}$ diameter) (Figure 2). These findings were compatible with brain abscess.

After eight days of medical treatment and observation with the patient was sent for stereotactic biopsy and aspiration of the abscess due to no neurological improvement. The procedure was done without complication and patient started showing signs of improvement. He became more alert and followed commands. Pathology reported gliotic brain tissue with organizing necrosis and hemorrhage without purulent inflammation. Fluid culture was negative.

Ten days after treatment with nafcillin, the patient presented with diffuse erythematous rash involving the palm of the hands. Biopsy of the lesions was remarkable for interface dermatitis, compatible with a drug eruption. Nafcillin was then switched to vancomycin $1 \mathrm{~g} \mathrm{q12hr}$. Patient remained stable and was transferred to medical floor and subsequently to rehabilitation

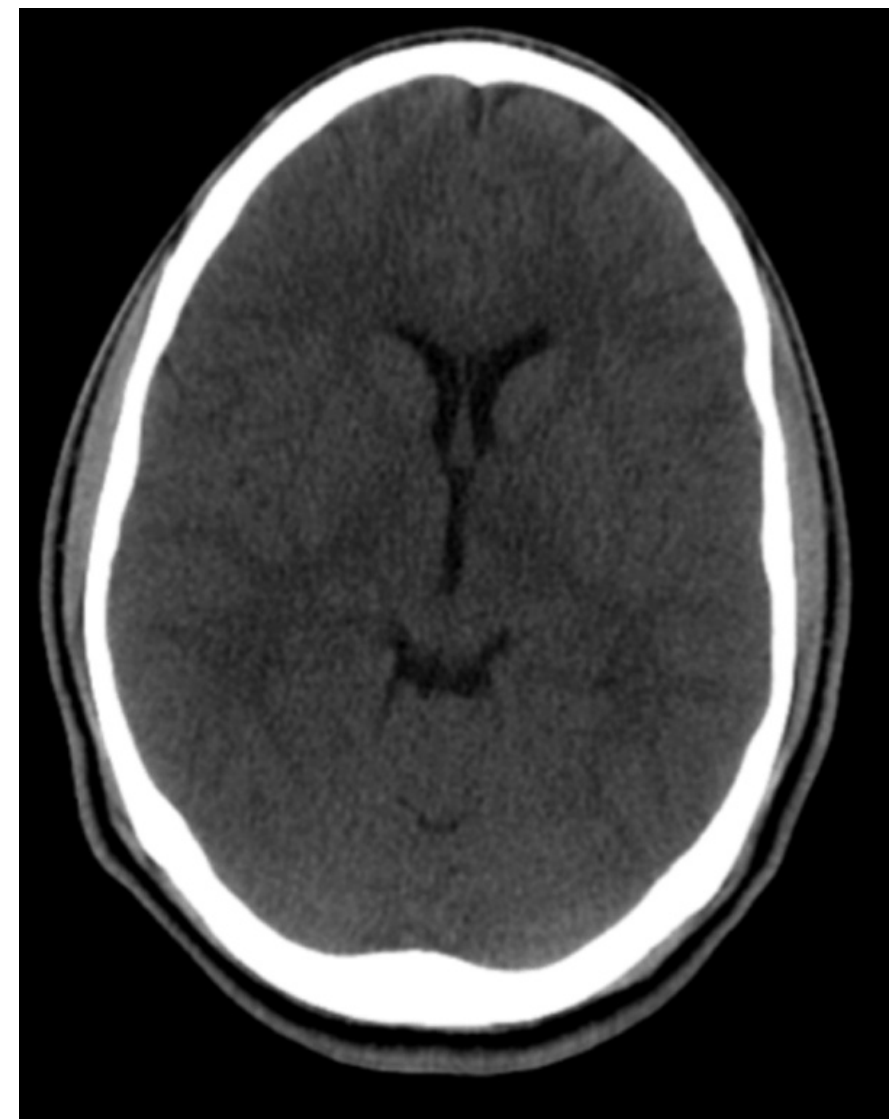

Figure 1: Non-contrast computed tomography scan of brain showing normal finding. 


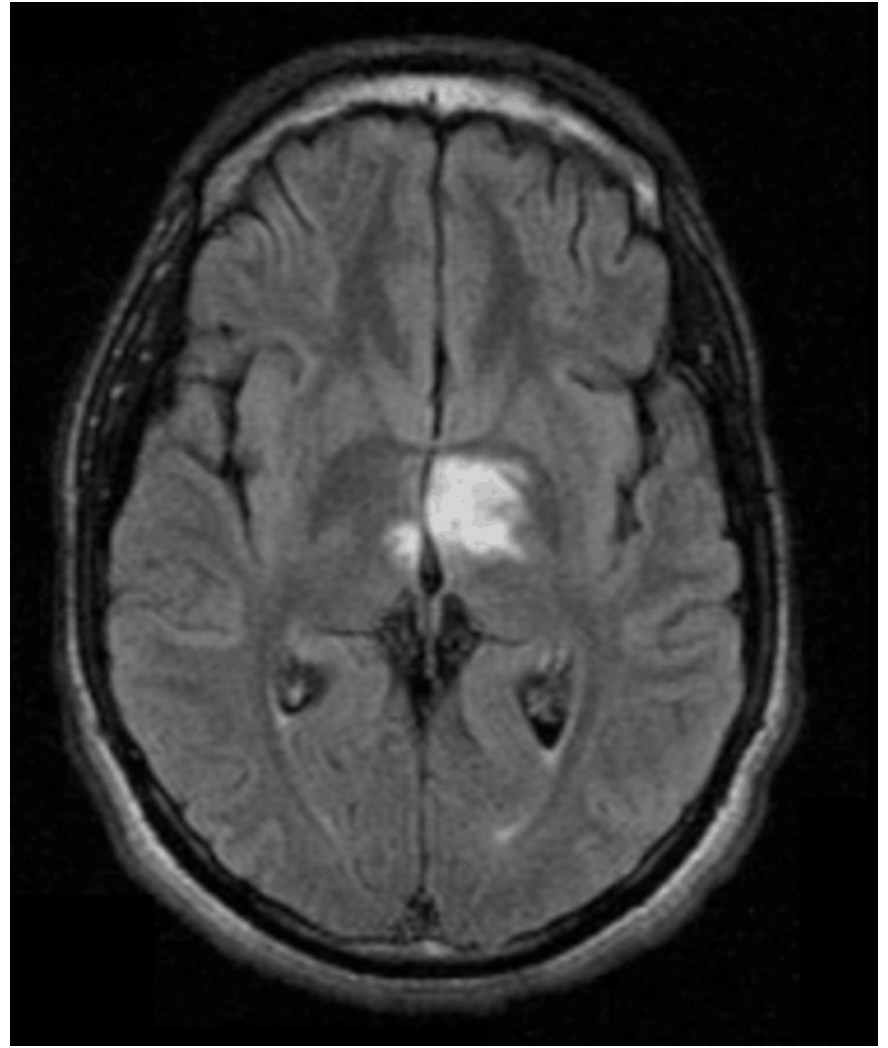

Figure 2: Brain MRI, Axial view showed a focal lesion centered at the left anterior thalamus with a small focus of contralateral extension into the right thalamus.

facility to continue antibiotic treatment for 6-8 weeks or until resolution of the brain abscess. Patient was seen four weeks after with complete neurological recuperation and resolution of the brain abscess on repeat MRI scan.

\section{DISCUSSION}

Staphylococcus aureus infections remains a major cause of morbidity and mortality, with presentations ranging from simple cutaneous infection to serious lifethreatening infections. Isolated meningitis caused by Staphylococcus aureus is rare accounting for less than $5 \%$ of all meningitis presentations [2]. On the other hand, Staphylococcus aureus is a major cause of cerebral abscess being the second most commonly found bacteria in patients with cerebral abscess, with Streptococcus milleri being the most common, and anaerobes the third most common [1, 3-5]. CNS infection caused by Staphylococcus aureus is usually associated with prior neurosurgical intervention or with typical risk factors such as endocarditis, soft tissue infection, skin abscesses, AIDS or intravenous drug use $[8,6]$. In this case, the only infection that could be associated with the MSSA bactermia was the mild suprapubic foliculitis, which we determined to be a very unlikely source as it was a mild cutaneous infection in an immunocompetent host. The patient was evaluated by oral maxillofacial surgery (OMS) department who determined that the prior dental work was an unlikely cause of the brain abscess and neurological infection that ensued.

The initial presentation of a patient with cerebral abscess could be very non-specific with only generalized symptoms. This makes the initial recognition very challenging. Different series have reported headache as the most common symptom in a patient with a brain abscess (41-63\% cases). Fever is an inconsistent finding, only reported in $25 \%$ cases [3, 5]. Altered mental status (AMS) and focal neurologic deficit are inconsistent findings reported in different series with a range for AMS being $18-48 \%$ and from $25-65 \%$ for focal neurologic deficit $[1,3-5]$. Other symptoms, especially gastrointestinal symptoms are present in about $27 \%$ cases [5]. In our patient, non-contrast CT scan of the head was performed and yielded a negative result on both presentations to the ED. A contrast MRI scan done several days after admission showed the thalamic abscess. The initial negative finding on CT scan and the absence of focal neurological deficit contributed to the delay in the diagnosis of brain abscess. Despite this, non-contrast CT scan is shown to be a good initial test for a patient with suspicion of cerebral abscess, even though in the early phase, non-contrast CT scan could be negative $[7,9,10]$. When it is used in combination with contrast CT scan, the sensitivity is very high, as was demonstrated in a series of 50 cases where positive CT scan finding was reported in $100 \%$ of the cases [1]. When CT scan is not diagnostic, MRI scan with contrast is in general the next step. Contrast MRI scan is highly accurate for detection of brain abscess [11].

Treatment of brain abscess is based on pertinent antibiotic therapy and neurosurgical mechanical drainage in most cases. Recommended initial empiric antibiotic treatment is a third generation cephalosporin in combination with metronidazole. If there is suspicion or risk factors for Staphylococcus aureus infection, vancomycin should be added until culture identification and sensitivities are available. Vancomycin remains the drug of choice when there is a suspicion for Staphylococcus aureus infection despite poor penetration in the CNS $[1-5,10]$. As a result, some authors have proposed linezolid as a good alternative for vancomycin, as it has better penetration in the CNS [12, $7,13]$. In general, if the abscess is more than $2-2.5 \mathrm{~cm}$, neurosurgical intervention is indicated. Stereotactic aspiration is the treatment of choice for abscesses located in the brain stem $[1,5,14]$.

\section{CONCLUSION}

Our case highlights the challenge of early diagnosis of brain abscess, an entity that presents very often with non-specific signs, the diagnosis of which could be easily missed, with repercussions on long-term disability and mortality. The mortality rate in patients with brain abscess remains high despite early detection and improvement in treatment. Long-term disability is also very high. Hence an early diagnosis and neurosurgical 
intervention could prove significant in the final outcome for these patients.

$* * * * * * * * *$

\section{Author Contributions}

Carlos Gonzalez - Substantial contributions to conception and design, Acquisition of data, Analysis and interpretation of data, Drafting the article, Revising it critically for important intellectual content, Final approval of the version to be published

Juan Roa - Substantial contributions to conception and design, Acquisition of data, Analysis and interpretation of data, Drafting the article, Revising it critically for important intellectual content, Final approval of the version to be published

Nehad Shabarek - Substantial contributions to conception and design, Acquisition of data, Analysis and interpretation of data, Drafting the article, Revising it critically for important intellectual content, Final approval of the version to be published

\section{Guarantor}

The corresponding author is the guarantor of submission.

\section{Conflict of Interest}

Authors declare no conflict of interest.

\section{Copyright}

(C) Carlos Gonzalez et al. 2013; This article is distributed under the terms of Creative Commons Attribution 3.0 License which permits unrestricted use, distribution and reproduction in any means provided the original authors and original publisher are properly credited. (Please see www.ijcasereportsandimages.com/copyright-policy.php for more information.)

\section{REFERENCES}

1. Lakshmi V, Rao RR, Dinakar I. Bacteriology of brain abscess--observations on 50 cases. J Med Microbiol 1993 Mar;38(3):187-90.

2. Roberts FJ, Smith JA, Wagner KR. Staphylococcus aureus meningitis: 26 years' experience at Vancouver General Hospital. Can Med Assoc J 1983 Jun 15;128(12):1418-20.

3. Sharma R, Mohandas K, Cooke RP. Intracranial abscesses: changes in epidemiology and management over five decades in Merseyside. Infection 2009 Feb;37(1):39-43.

4. Roche M, Humphreys H, Smyth E, et al. A twelveyear review of central nervous system bacterial abscesses; presentation and aetiology. Clin Microbiol Infect 2003 Aug;9(8):803-9.

5. Carpenter J, Stapleton S, Holliman R. Retrospective analysis of 49 cases of brain abscess and review of the literature. Eur J Clin Microbiol Infect Dis 2007 Jan;26(1):1-11.

6. Aguilar J, Urday-Cornejo V, Donabedian S, Perri M, Tibbetts R, Zervos M. Staphylococcus aureus meningitis: case series and literature reviewe. Medicine (Baltimore) 2010 Mar;89(2):117-25.

7. Naesens R, Ronsyn M, Druwé P, Denis O, Ieven M, Jeurissen A. Central nervous system invasion by community acquired meticillin-resistant Staphylococcus aureus. Journal of Medical Microbiology 2009;58(Pt 9):1247-51.

8. Pintado V, Meseguer MA, Fortún J, et al. Clinical study of 44 cases of Staphylococcus aureus meningitis. Eur J Clin Microbiol Infect Dis 2002 Dec;21(12):864-8.

9. Lo BM, Erwin EA. Missed epidural brain abscess after furunculosis. Am J Emerg Med. 2008 May;26(4):522.e3-4.

10. Muzumdar D, Jhawar S, Goel A. Brain abscess: An overview. Int J Surg 2011;9(2):136-44.

11. Haimes AB, Zimmerman RD, Morgello S, et al. MR imaging of brain abscesses. AJR Am J Roentgenol 1989;152(5):1073-85.

12. Rupprecht TA, Pfister HW. Clinical experience with linezolid for the treatment of central nervous system infection. Eur J nuerol 2005;12(7):536-42.

13. Saito N, Aoki K, Sakurai T, et al. Linezolid treatment for intracranial abscesses caused by methicillinresistant Staphylococcus aureus--two case reports. Neurol Med Chir (Tokyo) 2010;50(6):515-7.

14. Nishihara M, Sasayama T, Kudo H, Kohmura E. Morbidity of stereotactic biopsy for intracranial lesions. Kobe J Med Sci 2011 Jan 21;56(4):E148-53. 
Access full text article on

other devices

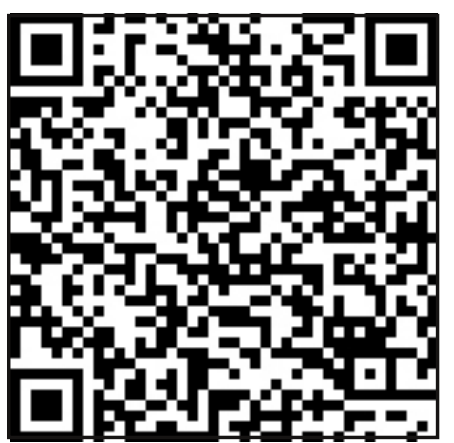

Access PDF of article on other devices

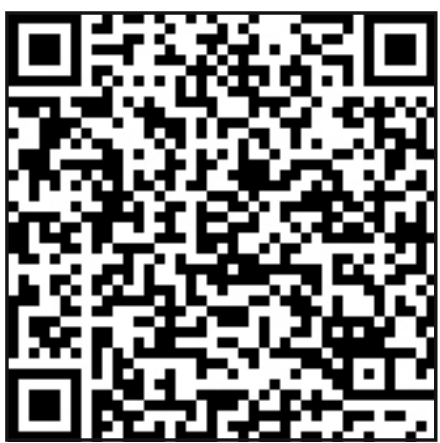

